\title{
Tabulation of Coefficients for Operations on Taylor Series
}

\author{
By Daniel C. Fielder
}

1. Introduction. When certain operations are performed on polynomials or power series, the results often assume the form of a series of ascendingly indexed terms. A typical term, the $k$ th, appears as the product of two distinct types of factors. One of these factors is a multiplier peculiar to the particular operation. The other factor is a group of literal coefficients which forms a $k$ th order symmetric function of weight $k$ and degree $h$ in those literal coefficients. A detailed examination reveals that the second factor is composed of sums of combinations of appropriately subscripted and exponentiated literal coefficients. This second factor, which will be called a literal group, has a form common to many operations on polynomials and power series. The multiplier of each combination within a group is observed to be either a positive integer or a positive common fraction.

As is well known, the weight of a term in a symmetric function is the sum of the products of corresponding subscripts and exponents. The degree is the sum of the exponents of a product combination of literal coefficients. As an example, for $k=6$ and $h=3$ the $k$ th term might be $\{6\}\left(g_{1}{ }^{2} g_{4}+[2] g_{1} g_{2} g_{3}+\left[\frac{1}{3}\right] g_{2}{ }^{3}\right)$. The weight of a product combination of literal coefficients, say the $g_{1}{ }^{2} g_{4}$ term, is $1 \times 2+$ $4 \times 1=6$, and the degree of a product combination, again using the $g_{1}^{2} g_{4}$ term, is $2+1=3$. For uniformity, the identification of enclosing symbols with algebraic terms used in the above example is used throughout the paper.

Several direct and inverse operations are investigated, and methods for finding general terms for groups and multipliers are discussed. For convenience, literal groups are tabulated. Because of the common properties of the literal groups, a single table of literal groups suffices for all operations described herein.

2. Power Series of Power Series. The first operation to be considered is

$$
\left(b_{1} y+b_{2} y^{2}+b_{3} y^{3}+\cdots\right)=\left(m_{1} x+m_{2} x^{2}+m_{3} x+\cdots\right),
$$

in which

$$
y=g_{1} x+g_{2} x^{2}+g_{3} x^{3}+\cdots .
$$

This is essentially 6.362 of Adams [1].

A direct calculation of the first few terms leads to

$$
\begin{aligned}
m_{1}= & \left\{b_{1}\right\}\left(g_{1}\right), \\
m_{2}= & \left\{b_{2}\right\}\left(g_{1}^{2}\right)+\left\{b_{1}\right\}\left(g_{2}\right), \\
m_{3}= & \left\{b_{3}\right\}\left(g_{1}^{3}\right)+\left\{2 b_{2}\right\}\left(g_{1} g_{2}\right)+\left\{b_{1}\right\}\left(g_{3}\right), \\
m_{4}= & \left\{b_{4}\right\}\left(g_{1}^{4}\right)+\left\{3 b_{3}\right\}\left(g_{1}^{2} g_{2}\right) \\
& +\left\{2 b_{2}\right\}\left(g_{1} g_{3}+\left[\frac{1}{2}\right] g_{2}^{2}\right)+\left\{b_{1}\right\}\left(g_{4}\right),
\end{aligned}
$$

Received November 24, 1959; revised April 25, 1960. 
Table 1 presents a general form for the literal and numerical (braced) coefficients up through index 12. For any $\left\{t_{k h}\right\}$ of Table 1 except $\left\{t_{k k}\right\}$, the numerical coefficients for the operation described by (1) and (2) are $h b_{h}$. For $\left\{t_{k k}\right\}$, the numerical coefficients are $b_{k}$.

TABLE 1. Values of literal groups, $k=1(1) 12$

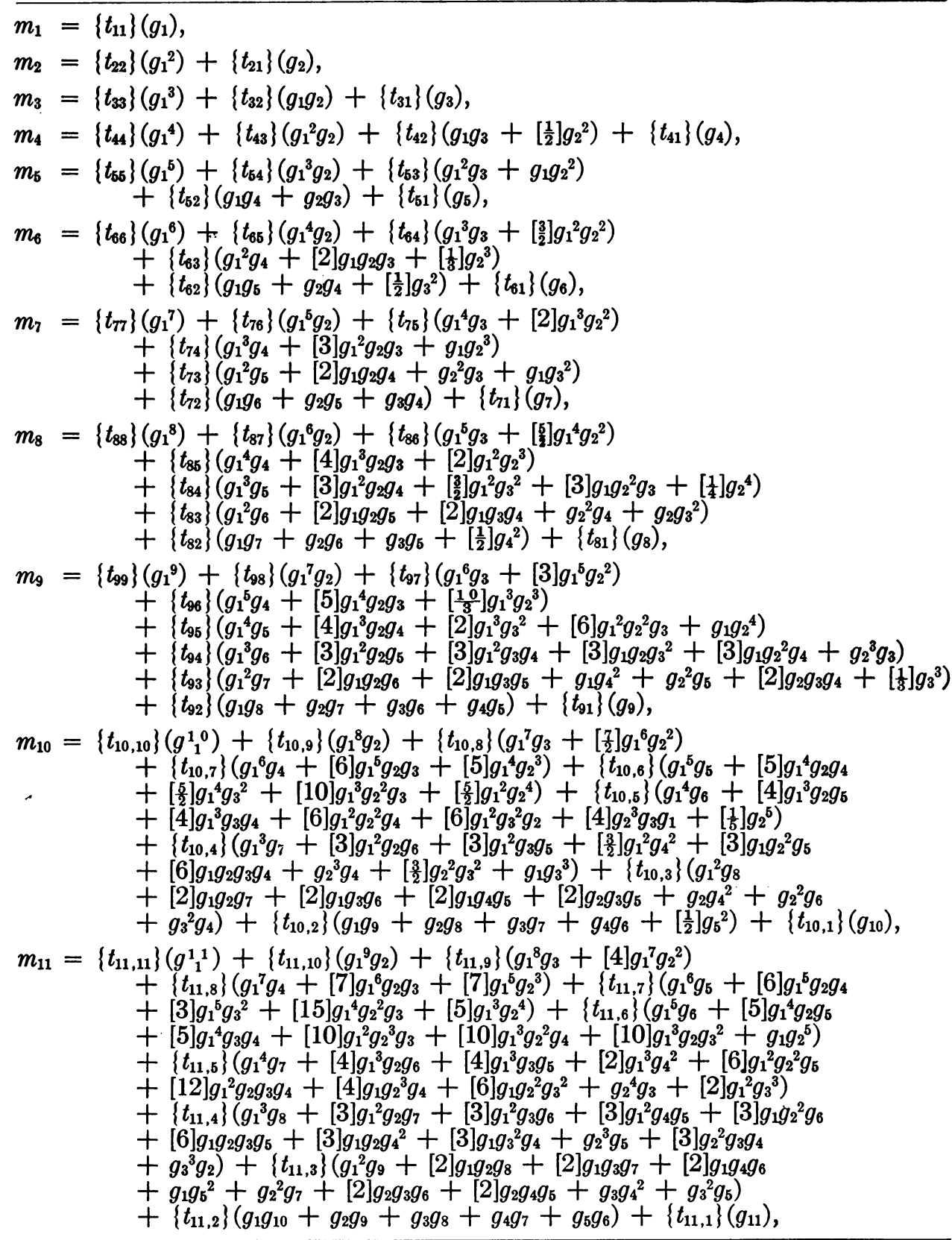


TABLE 1-Continued

$$
\begin{aligned}
& m_{12}=\left\{t_{12,12}\right\}\left(g^{12}{ }_{1}^{2}\right)+\left\{t_{12,11}\right\}\left(g_{1}{ }^{0} g_{2}\right)+\left\{t_{12,10}\right\}\left(g_{1}{ }^{9} g_{3}+\left[\frac{9}{2}\right] g_{1}{ }^{8} g_{2}{ }^{2}\right) \\
& +\left\{t_{12,9}\right\}\left(g_{1}^{8} g_{4}+[8] g_{1}^{7} g_{2} g_{3}+\left[\frac{28}{3}\right] g_{1}^{6} g_{2}^{3}\right)+\left\{t_{12,8}\right\}\left(g_{1}{ }^{7} g_{5}+\left[\frac{7}{2}\right] g_{1}{ }^{6} g_{3}{ }^{2}\right. \\
& \left.+[7] g_{1}^{6} g_{2} g_{4}+\left[{ }_{4}^{35}\right] g_{1}^{4} g_{2}^{4}+[21] g_{1}^{5} g_{2}{ }^{2} g_{3}\right)+\left\{t_{12,7}\right\}\left(g_{1}{ }^{6} g_{6}\right. \\
& +[3] g_{1}^{2} g_{2}^{6}+[6] g_{1}^{5} g_{2} g_{5}+[6] g_{1}^{5} g_{3} g_{4}+[15] g_{1}^{4} g_{2}^{2} g_{4}+[15] g_{1}{ }^{4} g_{2} g_{3}{ }^{2} \\
& \left.+[20] g_{1}{ }^{3} g_{2}^{3} g_{3}\right)+\left\{t_{12,6}\right\}\left(g_{1}^{5} g_{7}+\left[\frac{5}{2}\right] g_{1}{ }^{4} g_{4}^{2}+\left[\frac{10}{3}\right] g_{1}{ }^{3} g_{3}{ }^{3}+[5] g_{1}^{4} g_{2} g_{6}\right. \\
& +[5] g_{1}^{4} g_{3} g_{5}+[5] g_{1} g_{2}^{4} g_{3}+[10] g_{1}^{3} g_{2}^{2} g_{5}+[10] g_{1}^{2} g_{2}{ }^{3} g_{4} \\
& \left.+[15] g_{1}{ }^{2} g_{2}{ }^{2} g_{3}{ }^{2}+[20] g_{1}{ }^{3} g_{2} g_{3} g_{4}+\left[\frac{1}{6}\right] g_{2}{ }^{6}\right)+\left\{t_{12,5}\right\}\left(g_{1}{ }^{4} g_{8}+g_{2}{ }^{4} g_{4}\right. \\
& +[2] g_{2}{ }^{3} g_{3}{ }^{2}+[4] g_{1}{ }^{3} g_{2} g_{7}+[4] g_{1}{ }^{3} g_{3} g_{6}+[4] g_{1}{ }^{3} g_{4} g_{5}+[4] g_{1} g_{2}{ }^{3} g_{5} \\
& +[4] g_{1} g_{2} g_{3}^{3}+[6] g_{1}^{2} g_{2}^{2} g_{6}+[6] g_{1}^{2} g_{2} g_{4}^{2}+[6] g_{1}^{2} g_{3}^{2} g_{4} \\
& \left.+[12] g_{1}^{2} g_{2} g_{3} g_{5}+[12] g_{1} g_{2}^{2} g_{3} g_{4}\right)+\left\{t_{12,4}\right\}\left(g_{1}^{3} g_{9}+g_{2}^{3} g_{6}\right. \\
& +\left[\frac{3}{2}\right] g_{1}{ }^{2} g_{5}{ }^{2}+\left[\frac{8}{2}\right] g_{2}^{2} g_{4}^{2}+[3] g_{1}^{2} g_{2} g_{8}+[3] g_{1}^{2} g_{3} g_{7}+[3] g_{1} g_{2}{ }^{2} g_{7} \\
& +[3] g_{1}^{2} g_{4} g_{6}+[3] g_{1} g_{3}{ }^{2} g_{5}+[3] g_{1} g_{3} g_{4}{ }^{2}+[3] g_{2}{ }^{2} g_{3} g_{5} \\
& \left.+[3] g_{2} g_{3}^{2} g_{4}+[6] g_{1} g_{2} g_{3} g_{6}+[6] g_{1} g_{2} g_{4} g_{5}+\left[\frac{1}{4}\right] g_{3}^{4}\right) \\
& +\left\{t_{12,3}\right\}\left(g_{1}^{2} g_{10}+g_{2}^{2} g_{8}+g_{2} g_{5}^{2}+g_{3}^{2} g_{6}+[2] g_{1} g_{2} g_{9}+[2] g_{1} g_{3} g_{8}\right. \\
& +[2] g_{1} g_{4} g_{7}+[2] g_{1} g_{5} g_{6}+[2] g_{2} g_{3} g_{7}+[2] g_{2} g_{4} g_{6}+[2] g_{3} g_{4} g_{5} \\
& \left.+\left[\frac{1}{3}\right] g_{4}^{3}\right)+\left\{t_{12,2}\right\}\left(g_{1} g_{11}+g_{2} g_{10}+g_{3} g_{9}+g_{4} g_{8}+g_{5} g_{7}+\left[\frac{1}{2}\right] g_{6}{ }^{2}\right) \\
& +\left\{t_{12,1}\right\}\left(g_{12}\right) \text {. }
\end{aligned}
$$

3. Reversion of Power Series. (See 6.26 of Adams [1] and Van Orstrand [2].) If

$$
z=x-g_{1} x^{2}-g_{2} x^{3}-g_{3} x^{4}-\cdots
$$

is reversed to yield

$$
x=z+m_{1} z^{2}+m_{2} z^{3}+m_{3} z^{4}+\cdots,
$$

the result is similar to that noted in the preceding section. The first few terms are

$$
\begin{aligned}
m_{1}= & \{1\}\left(g_{1}\right), \\
m_{2}= & \{2\}\left(g_{1}^{2}\right)+\{1\}\left(g_{2}\right), \\
m_{3}= & \{5\}\left(g_{1}^{3}\right)+\{5\}\left(g_{1} g_{2}\right)+\{1\}\left(g_{3}\right), \\
m_{4}= & \{14\}\left(g_{1}^{4}\right)+\{21\}\left(g_{1}^{2} g_{2}\right) \\
& +\{6\}\left(g_{1} g_{3}+\left[\frac{1}{2}\right] g_{2}^{2}\right)+\{1\}\left(g_{4}\right),
\end{aligned}
$$

Table 1 applies as before. For any $\left\{t_{k h}\right\}$ of Table 1 except $\left\{t_{k k}\right\}$, the numerical coefficients for the operation described by (4) and (5) are the familiar binomial coefficients $\left(\begin{array}{l}k+h \\ k+1\end{array}\right)$. For $\left\{t_{k k}\right\}$, the numerical coefficients are $\left[\left(\begin{array}{c}2 k \\ k\end{array}\right)-\left(\begin{array}{c}2 k \\ k-1\end{array}\right)\right]$.

4. Development of a Power Series Ratio. The four succeeding operations are based on a tabulation derived from the ratio of two power series. A short discussion of this tabulation follows. The two power series and the quotient $P(z)$ are

$$
\frac{\alpha_{0}+\alpha_{1} z+\alpha_{2} z^{2}+\cdots}{\beta_{0}+\beta_{1} z+\beta_{2} z^{2}+\cdots}=P(z)=C_{0}+C_{1} z+C_{2} z^{2}+\cdots .
$$


If the indicated division is performed, a triangular tabulation array can be obtained as shown below.

$$
\begin{aligned}
& C_{0} \beta_{0}-\alpha_{0}=0 \\
& C_{1} \beta_{0}+C_{0} \beta_{1}-\alpha_{1}=0 \\
& C_{2} \beta_{0}+C_{1} \beta_{1}+C_{0} \beta_{2}-\alpha_{2}=0 \\
& C_{3} \beta_{0}+C_{2} \beta_{1}+C_{1} \beta_{2}+C_{0} \beta_{3}-\alpha_{3}=0
\end{aligned}
$$

Thus, if the $\alpha$ 's and $\beta$ 's of the array (8) are known, the $C$ 's can be found by the division indicated in (7).

5. Direct and Inverse Summation Formulas of Power of Roots. In a previous paper, the author [3] discusses means of finding sums of powers of roots of the integral rational function

$$
f(x)=a_{0} x^{n}+a_{1} x^{n-1}+\cdots+a_{n-1} x+a_{n}
$$

by elaborating on a method credited to Newton [1]. Newton's method utilizes the tabulation,

$$
\begin{aligned}
& 1 a_{1}+S_{1} a_{0}=0, \\
& 2 a_{2}+S_{1} a_{1}+S_{2} a_{0}=0, \\
& 3 a_{3}+S_{1} a_{2}+S_{2} a_{1}+S_{3} a_{0}=0,
\end{aligned}
$$

where the sum of the $b$ th powers of the roots of $f(x)$ are

$$
S_{b}=r_{1}^{b}+r_{2}^{b}+r_{3}^{b}+\cdots \text {. }
$$

A comparison of (8) and (10) suggests that it should be possible to find a $P(z)$ whose coefficients are functions of the $a$ 's of (10) and are simply related to $S_{k}$ 's. Such is indeed the case for the determination of the direct summation formulas. For convenience, (10) is rearranged and restated as

$$
\begin{aligned}
& m_{1}+g_{1}=0, \\
& 2 m_{2}+m_{1} g_{1}+2 g_{2}=0, \\
& 3 m_{3}+2 m_{2} g_{1}+m_{1} g_{2}+3 g_{3}=0 ;
\end{aligned}
$$

where $g_{k}=\left(a_{k} / a_{0}\right)$ and $m_{k}=\left(S_{k} / k\right)$.

For the direct summation, it is seen that $\beta_{0}=1, C_{k}=(k+1) m_{k+1}, \beta_{k}=g_{k}$, and $\alpha_{k}=-k g_{k}$. Thus, $P(z)$ becomes

$$
P(z)=\frac{-g_{1}-2 g_{1} z-3 g_{3} z^{2}-4 g_{4} z^{3} \cdots}{1+g_{1} z+g_{2} z^{2}+g_{3} z^{3}+\cdots} .
$$

The first few terms of the indicated division are

$$
\begin{aligned}
& -g_{1}+\left(g_{1}^{2}-2 g_{2}\right) z+\left(-g_{1}^{3}+3 g_{1} g_{2}-3 g_{3}\right) z^{2} \\
& +\left(g_{1}^{4}-4 g_{1}^{2} g_{2}+4 g_{1} g_{3}+2 g_{2}^{2}-4 g_{4}\right) z^{3}+\cdots,
\end{aligned}
$$


where $-g_{1}=C_{0}, g_{1}^{2}-2 g_{2}=C_{1}$, etc. Thus,

$$
\begin{aligned}
m_{1}= & \{-1 / 1\}\left(g_{1}\right), \\
m_{2}= & \{1 / 2\}\left(g_{1}^{2}\right)+\{-1\}\left(g_{2}\right), \\
m_{3}= & \{-1 / 3\}\left(g_{1}^{3}\right)+\{1\}\left(g_{1} g_{2}\right)+\{-1\}\left(g_{3}\right), \\
m_{4}= & \{1 / 4\}\left(g_{1}^{4}\right)+\{-1\}\left(g_{1}^{2} g_{2}\right) \\
& +\{1\}\left(g_{1} g_{3}+[1 / 2] g_{2}^{2}\right)+\{-1\}\left(g_{4}\right),
\end{aligned}
$$

It is apparent that (13) not only yields the desired summations but serves as a device for generating the literal groups of Table 1. For any $\left\{t_{k r}\right\}$ of Table 1 except $\left\{t_{k k}\right\}$, the numerical coefficients for the direct summation are $(-1)^{h}$. For $\left\{t_{k k}\right\}$, the numerical coefficients are $(-1)^{k} / k$.

It is not possible to use (12) directly to find a $P(z)$ whose coefficients lead to the inverse summation forms. However, a two-step solution leading to the inverse summation forms can be found by rearranging (12) as

$$
\begin{aligned}
& g_{1}+B_{1}=0, \\
& g_{2}+m_{1} g_{1}+2 B_{2}=0, \\
& g_{3}+m_{1} g_{2}+2 m_{2} g_{1}+3 B_{3}=0,
\end{aligned}
$$

where

$$
\begin{aligned}
& 1 B_{1}=1 m_{1}+0 g_{1}, \\
& 2 B_{2}=2 m_{2}+1 g_{2}, \\
& 3 B_{3}=3 m_{3}+2 g_{3},
\end{aligned}
$$

The relationships $g_{k}=\left(a_{k} / a_{0}\right)$ and $m_{k}=\left(S_{k} / k\right)$ apply as before.

For the inverse summation, it is seen that $\beta_{0}=1, C_{k}=g_{k}, \beta_{k}=k m_{k}$, and $\alpha_{k}=(k+1) B_{k+1}$. Thus, the $P(z)$ becomes

$$
\begin{aligned}
P(z) & =\frac{-B_{1}-2 B_{2} z-3 B_{3} z^{2}-4 B_{4} z^{3}-\cdots}{1+m_{1} z+2 m_{2} z^{2}+3 m_{3} z^{3}+\cdots} \\
& =g_{1}+g_{2} z+g_{3} z^{2}+g_{4} z^{3}+\cdots
\end{aligned}
$$

The first few terms are

$$
-B_{1}+\left(B_{1} m_{1}-2 B_{2}\right) z+\left(-B_{1} m_{1}^{2}+2 B_{2} m_{1}+2 B_{1} m_{2}-3 B_{3}\right) z^{2}+\cdots .
$$

Through use of (17), the $B$ terms are eliminated and the result is

$$
\begin{aligned}
g_{1}= & \{-1 / 1 !\}\left(m_{1}\right), \\
g_{2}= & \{1 / 2 !\}\left(m_{1}^{2}\right)+\{-1 / 0 !\}\left(m_{2}\right), \\
g_{3}= & \{-1 / 3 !\}\left(m_{1}^{3}\right)+\{1 / 1 !\}\left(m_{1} m_{2}\right)+\{-1 / 0 !\}\left(m_{3}\right), \\
g_{4}= & \{1 / 4 !\}\left(m_{1}^{4}\right)+\{-1 / 2 !\}\left(m_{1}{ }^{2} m_{2}\right) \\
& +\{1 / 1 !\}\left(m_{1} m_{3}+[1 / 2] m_{2}{ }^{2}\right)+\{-1 / 0 !\}\left(m_{4}\right), \\
& \ldots \ldots \ldots \ldots \ldots \ldots \ldots \ldots \ldots \ldots \ldots \ldots \ldots \ldots \ldots \ldots \ldots \ldots \ldots \ldots \ldots \ldots \ldots \ldots \ldots \ldots
\end{aligned}
$$


Except for different numerical coefficients in braces and the interchange of the roles of the $m$ 's and $g$ 's, (20) is identical with (15). The discussion following (15) thus applies for finding the literal groups of (20). Table 1 is immediately adaptable with appropriate changes of notation. For any $\left\{t_{k k}\right\}$ of Table 1 except $\left\{t_{k k}\right\}$, the numerical coefficients for the inverse summation are $(-1)^{h} /[(k+h)-(k+1)]$ !. For $\left\{t_{k k}\right\}$, the numerical coefficients are $(-1)^{k} / k$ !.

6. Logarithms and Exponentials of Power Series. Expression 6.364 of Adams [1] is essentially

$$
\ln \left(1+g_{1} z+g_{2} z^{2}+g_{3} z^{3}+\cdots\right)=m_{1} z+m_{2} z^{2}+m_{3} z^{3}+\cdots
$$

Through a comparison of Adams' table accompanying 6.364 with (8) and (12) and an application of the reasoning of the last section, the arrangement of the $m$ 's and $g$ 's of $(21)$ becomes that of Table 1 . For any $\left\{t_{k k}\right\}$ of Table 1 except $\left\{t_{k k}\right\}$, the numerical coefficients are $(-1)^{h+1}$. For $\left\{t_{k k}\right\}$, the numerical coefficients are $(-1)^{k+1} / k$.

The exponential form is given as

$$
\exp \left(g_{1} z+g_{2} z^{2}+g_{3} z^{3}+\cdots\right)=1+m_{1} z+m_{2} z^{2}+m_{3} z^{3}+\cdots .
$$

Again through use of the methods of the last section, the arrangement of the $m$ 's and $g$ 's becomes that of Table 1 . For any $\left\{t_{k k}\right\}$ of Table 1 except $\left\{t_{k k}\right\}$, the numerical coefficients for the exponential form are $1 /[(k+h)-(k+1)]$ !. For $\left\{t_{k k}\right\}$, the numerical coefficients are $1 / k !$.

7. Calculation of Numerical Coefficients within a Literal Group. It becomes apparent that in any literal group except those which are coefficients of the $\left\{t_{k k}\right\}$ 's the term $g_{1}^{h-1} g_{k-h+1}$ always appears. This term has weight $k$, degree $h$, and multiplier unity. The letter combinations in any literal group consist of all the combinations of $g$ 's which simultaneously have weight $k$ and degree $h$. The quotient formed by $(h-1)$ ! divided by the products of the factorials of the exponents of a combination of $g$ 's determines the bracketed numerical coefficient for that combination of $g$ 's. The single term of the $g_{1}{ }^{k}$ group (i.e., the coefficient of $\left\{t_{k k}\right\}$ ) has a multiplier of unity. This method for finding the terms of a literal group is essentially that outlined in Fielder [3].

8. Conclusions. It can be concluded that there are algebraic operations on polynomials or power series which have common forms for the literal groups. The braced coefficients are peculiar to particular algebraic operations. For a given operation, all numerical coefficients except $\left\{t_{k k}\right\}$ follow one combinatorial rule, while the $\left\{t_{k k}\right\}$ coefficients follow a different combinatorial rule. Since available tables of many algebraic operations which follow patterns similar to those discussed herein are terminated at index four or five, the information contained herein should be useful in extending the available tables. It is anticipated that 
many other algebraic operations on polynomials can be found which are members of the operation class of this paper.

Georgia Institute of Technology

Atlanta, Georgia

1. E. P. Adams, Smithsonian Mathematical Formulae and Tables of Elliptic Functions, Smithsonian Institution, Washington, 1947.

2. C. E. VAN Orstrand, "Reversion of power series," Phil. Mag., 6th Series, v. 19, 1910, p. $366-376$.

3. D. C. FIELDER, "A note on summation formulas of powers of roots," MTAC v. 12, July, 1958, p. 194-198. 